\title{
CLASS III MALOCCLUSION: A PERSONAL PERSPECTIVE
}

Kerr WJS. Class III malocclusion: a personal perspective. Annals Dent Univ Malaya 1996; 3: 23-26

\begin{abstract}
The aetiology and treatmont of Class III malocclusion characterised by lingual occlusion of the maxillary incisors are discussed in relation to patients in the West of Scotland. Mandibular size and position are identlfied as being the principal skeletal causes of an adverse relationship.

Treatment rationale is relatsd to the severity of the condition, but early diagnosis is important as the treatment may be considerably simplified. A range of appliances can be employed from upper removable, to quadhelix, to FRIII, to full upper and lower fixed appliances, depending upon the severity and the presenting features. As a "rule of thumb" -4 degrees ANB can be considered as the borderline between a purely orthodontic and a combined surgical approach.
\end{abstract}

Key Words: Class III malocclusion, Orthodontic appliances
Class III malocclusion: a personal perspective

\section{W John S Kerr}

Professor of Orthodontics

University of Glasgow

Department of Child Dental Care Glasgow

Dental Hospital and School

378 Sauchiehall Street

Glasgow G2 3JZ

Scotland, U.K.

Address for correspondence

Professor W J S Xerr, Glasgow Dental Hospital and School, 378

Sauchiehall Street, Glasgow G2 3JZ, Scotland, United Kindom.

\section{Introduction}

Class III malocclusion is a relatively uncommon occlusal relationship in the United Kingdom, being present in $3-4 \%$ (1) of the population. In the West of Scotland, however, the condition has more than double this prevalence (2) and is therefore of particular interest in the Glasgow area.

Although a range of morphological types theoretically occurs in this malocclusion group (3), ranging from those presenting with a small maxilla, a normal mandible and an increased Frankfort-mandlbular planes' angle, to those characterised by a normal maxilla, a prognathic mandible and a low Frankfort-mandibular planes' angle, in practice such pure morphological types are seldom encountered. Occlusally, Class III incisor relation, unlike that in the other malocclusion groups, has three possible definitions:

i. lower incisor edges biting anterior to the cingulum plateau of the upper incisors

ii. edge to edge relationship with overbite/overjet $=0 \mathrm{~mm}$

iii. negative overjet

The last is the most severe and consequently the most difficult to treat and is the subject of this paper.

\section{Aetiology}

A skeletal Class III relationship characterised by an ANB angle of $<2$ degrees is the product of a number of interrelated components. The contribution of each component may vary from case to case and from racial group to racial group.

The sample group which is the subject of the discussion below comprised of 31 boys randomly drawn from records at the Glasgow Dental Hospital and School and representing each of the 4 Angle malocclusion groups. The mean age of the subpcts was 10.4 years. For inclusion in the study (4) Class III subjects must have 11,12 in lingual occlusion. They were therefore marked Class III malocclusions and exhibited a mean ANB angle of -2 degrees (see Table 1 ).
Table 1. Cephalometric variables for boys aged 10.4 years in oach of the major malocclusion groups $(\mathrm{N}=31)$

\begin{tabular}{|l|r|r|r|r|}
\hline \multicolumn{5}{|c|}{ Angle Classs } \\
\hline Variable & \multicolumn{1}{|c|}{ II $_{1}$} & \multicolumn{1}{c|}{ II $_{2}$} & \multicolumn{1}{c|}{ I } & \multicolumn{1}{c|}{ III } \\
\hline SNA $^{0}$ & 80.5 & 79.9 & 80.8 & 79.9 \\
SNB $^{0}$ & 73.9 & 75.9 & 78.1 & 81.9 \\
ANB $^{0}$ & 6.6 & 4.0 & 2.7 & -2.0 \\
S-N(mm) & 72.0 & 71.6 & 71.0 & 69.6 \\
Ba-N(mm) & 108.8 & 106.7 & 105.9 & 102.3 \\
Ba-S-N & 130.3 & 126.9 & 127.1 & 123.2 \\
Co-Gn(mm) & 107.6 & 107.4 & 111.2 & 109.7 \\
ANS-Me(mm) & 65.7 & 62.8 & 65.5 & 62.2 \\
\hline
\end{tabular}

It was notable that, whereas there was a significant difference between all the groups with respect to angle SNB, there was no significant difference in angle SNA. Maxillary retrognathism was therefore not a feature of this population group.

\section{Aetiological components}

1. Mandibular size

The length of the mandible (Co-Gn) in Class III malocclusion was greater than that in both divisions of Class II but not significantly larger than that in Class I ( Table 1).

Consequently in this group mandibular size alone was not the only aetiological component in the Class III skeletal relationship.

2. Mandibular position

i. Antero-posterior

The mandibular condyle articulates with the glenoid fossa, which in turn is related to the posterior portion of the mid-line cranial base. The mornhology of the cranial hase therefore will 
determine the antero-posterior position of the condyle with respect to the profile.

In this group it is seen that both the anterior cranial base (S-N) and cranial base angle (Ba-S-N) are smallest in the Class III group contributing, overall, to a smaller effective length of the cranial base (BaN) - see Table 1. It is further important to realize that the shape and size of the anterior cranial base alters very little after 7 years $(5,6)$ although growth of the posterior cranial base continues into the teens. Anterior displacement of the mandible is considered to be a common occurrence in Class III malocclusion where there is a negative overjet (7). Although this view is widely accepted there is little scientific evidence to support the concept. Gravely (8) examined the lateral skull radiographs of 50 subjects with Class III malocclusions characterised by negative overjet and with suspected anterior displacment of the mandible, based on clinical examination. These subjects had had films taken both in maximum intercuspation of the posterior teeth and with the anterior teeth edge to edge. In the former radiograph angle ANB was -5 degrees and in the latter -3 degrees. He then formed a template from the mandible on the maximum intercuspation film and rotated it until the teeth were edge to edge. If anterior displacement of the mandible existed this would represent a position anterior to the edge to edge film. No statistically significant difference was found. He therefore could not support the hypothesis of anterior displacement in these cases, but suggested that it may be more common where the overbite is shallow and where the residual displacement had not been dissipated by further closure of the mandible.

ii. Vertical

To verify the hypothesis of Gravely, Kerr and TenHave (9) examined the lateral skull radiographs of 66 cases with lingual occlusion of 11,12 which had been treated orthodontically with a variety of appliances. Measurements were made to record the vertical and horizontal changes in mandibular position during treatment and these were compared with an untreated control group of comparable age and sex. If anterior displacement of the mandible was a common occurrence the mandible should move distally relative to the control group once it is eliminated as a result of treatment. When the two groups were compared, whilst all 5 vertical measurements increased significantly more in the appliance group during the period of study, only one horizontal distance was significantly less in the appliance group at the $\mathrm{p}<0.05$ level (CondylionPterygo-maxillary vertical). Measurement error showed that the two landmarks concerned (a point and a plane) were amongst those with the highest method error and this result should therefore be treated with caution. It would appear from this study, therefore, that overclosure of the mandible in this type

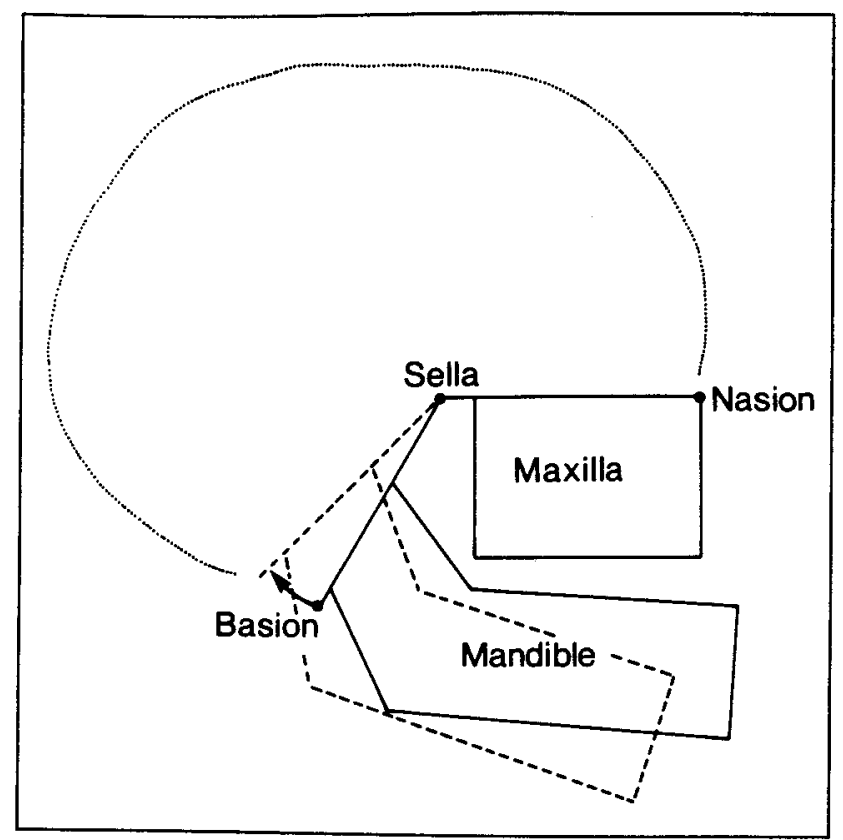

Figure 1: Diagramatic representation of the effect of cranial base morphology on mandibular prognathism. A longer, more obsusely angled, cranial base reduces mandibular prognathism in two subjects with mandibies of similars size..

of Class III malocclusion is more common than anterior displacement. Consequently the more overclosed the mandible the more prognathic it becomes. A shorter vertical dimension (ANS-Me) was also demonstrated in the cases cited in the first study (4). Figure 1 demonstrates the effect of cranial base morphology and size and mandibular position in influencing mandibular prognathism. Although maxillary retrognathism is not a common feature of the study population, when it occurs it will compound the effects described above.

\section{Treatment}

The basic objective in the orthodontic correction of Class III malocclusion is to compensate for the skeletal discrepancy by expansion of the upper arch and contraction of the lower. The prognosis for orthodontic correction of the type of Class III malocclusion discussed above, therefore, depends upon:

i. The degree of skeletal discrepancy.

The more severe the poorer the prognosis. Neverthelesss the shorter the vertical dimension the greater is the potential for posterior rotation of the mandible during treatment. In this context the age of tho patient will also be important as there is a tendency for the ANB angle, on average, to become more negative with growth and increasing age.

ii. The degree of overbite.

The corrected incisor relationship will depend upon the overbite for stability and therefore the greater it is initially the more favourable will be the prognosis.

iii. The incisor inclinations.

To some extent these reflect the degree of antero-posterior discrepancy. Within certain limits, the 
greater the discrepancy the more proclined the upper incisors and the more retroclined the lowers. As orthodontic appliances principally alter the inclinations of the incisors in Class III treatment the more proclined the upper incisors and the more retroclined the lowers the worse the prognosis.

iv. Crowding

Again crowding reflects the nature of the skeletal discrepancy and is therefore more common in the upper arch. Consequently the more crowding in the upper and the more spacing in the lower the worse the prognosis. Crowding in the lower arch may be regarded as a favourable prognostic sign as it indicated a potential for contraction of the lower arch perimeter following extraction.

\section{Rationale}

For the purpose of discussion Class III malocclusions can be divided into three groups on the basis of their degree of skeletal discrepancy:

1. Mild

These subjects should be identified as early as possible in the mixed dentition and are normally amenable to treatment with $a_{\text {; }}$ simple upper removable appliance bearing a posterior bite plane where the overbite is more than $2 \mathrm{~mm}$. Once the upper central incisors have erupted treatment can be initiated and normally takes only a few months. Where there is sufficient space, erupting lateral incisors will often be carried with the central incisors as they move labially. It is important to remember that anterior movement of the upper incisors represents expansion of the arch and will therefore create additional space which may be sufficient to treat mild degrees of crowding. Extraction of the lower deciduous canines may be helpful in allowing some resolution of lower crowding by relative liugual movement of the lower incisors. Extraction of the upper deciduous canines may have to be undertaken where the upper crowding prevents forward movement of the upper lateral incisors. In many cases this is all the treatmont which will be required except for that of crowding and local anomalies, which may be dealt with in the early permanent dentition. As an upper removable appliance will produce about 2.5 degrees of proclination for every $1 \mathrm{~mm}$ increase in overjet (10) the initial inclination of the upper incisors should be such that this is acceptable.

\section{Moderate}

This is probably the most problematic group of Class III malocclusions as many of them will be on the borderline between orthodontics and surgery.

Nevertheless a considerable number are amenable to orthodontic correction.

There are therefore a number of approaches:

a) Treat as above with a removable appliance and monitor. As this treatment is of short duration there is little to be lost.

b) Where the anterior crossbite is combined with a posterior crossbite, indicating a skeletal discrepancy in the lateral as well as the a-p dimension, a quadhelix appliance, banded to 16,26 can be employed. This should also be üsed early and active treatment is also of short duration.

Anterior helices can be added to correct anterior crossbites or the crossbites can be treated in two stages using a removable appliance for the anterior crossbite followed by a quadhelix.

If the posterior crossbite is confined to one molar an expansion screw can be added to the removable appliance used for proclination of the incisors and both crossbites treated simultaneously.

c) Where the antero-posterior discrepancy is more severe and marked anterior movement of the upper incisors is contraindicated, the maximum benefit is provided in the mixed dentition child with the FRIII of Frankel.

As with those cases cited above a good overbite is essential for success with this approach. The appliance will alter both upper and lower incisor inclinations sparingly, at the same time altering the posture of the mandible by backward rotation to a greator extent than an upper removable or upper and lower fixed appliances (10).

d) Delay treatment until the early permanent dentition and treat with fixed appliances. This approach may be indicated where the prognosis is less good and there are other identifiable features of malocclusion such as an impacted maxillary canine or rotations which will require fixed appliances for correction. The general principle is to produce alignment of the arches with/without extraction, as appropriate, and use Class III inter-maxillary traction to correct the incisor crossbite.

\section{Severe}

Even if the above protocol is followed relapse may still occur where growth is unfavourable. Consequently it is pointless to retain a corrected Class III incisor relationship for more than six months in an adolescent or pre-adolescent individual where the pattern of growth is unfavourable. In such cases surgery may have to be contemplated once growth has ceased, combined with orthodontic decompensation to correlate the dental arches in a Class I relationship. Severe Class III malocclusions, which are identified early, should be monitored with cephalometric control so that growth trends can be identiffed, as well as the cessation of growth.

When is a case severe enough to be beyond orthodontic correction? Opinion is somewhat subjective and will depend upon a number of 
factors, not least the patient's aesthetic requirements and the cultural context.

A recent study of adult Class III cases treated both by surgery combined with orthodontics and orthodontic correction alone, was carried out on patients from the West of Scotland (11). Analysis of the pre-treatment lateral skull radiographs showed two variables to distinguish between the groups with cut off points for ANB at -4 degrees and the inclination of the lower lncisor to the mandibular plane at 83 degrees. These values represented the subjective assessments of tho clinicians concerned but may be of use as guidelines in making the "surgery or orthodontics?" decision.

Where maxillary retrognathism of hypoplasia is a feature of the malocclusion, such as in cleft palate cases reverse headgear may also be considered.

\section{Conclusions}

1. The aetiology of Class III maloclusion with crossbite of 11,21 is the product of mandibular size and mandibular position, both antero-posteriorly and vertically.

2. A good prognosis for orthodontic correction is dependent upon a mild degree of skeletal discrepancy, a deep overbite, incisor inclinations which are near normal and crowding of the lower rather than the upper arch.

3. Early diagnosis facilitates optimum treatment and all but the most severe cases can be offered treatment in the mixed dentition.

4. The rationale for the different treatment modalities is discussed but the effects of all orthodontic appliances are confined to the alteration of incisor inclinations and the backward rotation of the mandible to differing degrees.

5. An ANB discrepancy of -4 degrees can be used as a guide for the borderline between orthodontic and surgical correction.

\section{Refences}

1) For TD, Walpole Day AJ. A survey of malocclusion and the need for orthodontic treatment in a Shropshire school population. Br J Orthod 1974; 1:73-5.

2) Luffingham JK, Campbell HM. The need for orthodontic treatment. A pilot survey of 14 year old school children in Paisley, Scotland. Trans European Orthodontic Society 1974; 259-67.

3) McCallin SG. Angle's Class III malocclusion. Dent Practit 1956; 6:151-64.

4) Kerr WJS, Adams CP. Cranial base and jaw relation. Am J Phys Anthrop 1988; 77:213-20.

5) de Coster L. A new line of reference for the study of lateral facial teleradiographs. Am J Orthod 1953; 39:304-6.

6) Kerr WJS. A method of superimposing lateral skull radlographs for the purpose of comparison. Br J Orthod 1978; 5:51-3.

7) Houston WJB, Tulley J. A textbook of orthodontics. Wright, Bristol, 1986; 3:27.

8) Gravely JF. A study of the mandibular closure path in Angle's Class III relationship. Br J Orthod 1984; 11:85-9.

9) Kerr WJS, TenHave TR. Mandibular position in Class III malocclusion. Br J Orthod 1988a; 15:241-5.

10) Kerr WJS, TenHave TR. A comparsion of Class III malocclusions treated by three appliance systems. Eur J Orthod 1988b; 10:266-80.

11) Kerr WJS, Miller S, Dawber J. Class III malocclusion: surgery or orthodontics? Br J Orthod 1992; 19:21-4. 